EPJ Web of Conferences 60, 20047 (2013)

DOI: $10.1051 /$ epjconf $/ 20136020047$

(C) Owned by the authors, published by EDP Sciences, 2013

\title{
Searching for intrinsic charm in the proton at the LHC
}

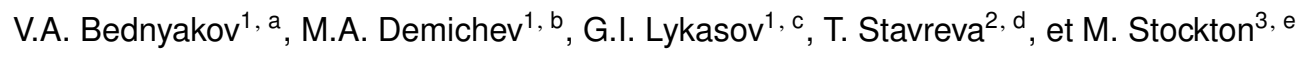 \\ ${ }^{1}$ Joint Institute for Nuclear Research, Dubna 141980, Moscow region, Russia \\ ${ }^{2}$ Laboratoire de Physique Subatomique et de Cosmologie, UJF, CNRS/IN2P3,INPG, 53 avenue des Martyrs, 38026 Grenoble, \\ France \\ ${ }^{3}$ Department of Physics, McGill University, Montreal QC, Canada
}

\begin{abstract}
The LHC with $p p$-collisions at $\sqrt{s}=7-14 \mathrm{TeV}$ will obviously supply extra unique information concerning the non-zero intrinsic (or valence-like) heavy quark component of the proton distribution functions component of the proton. On the basis of theoretical study it is demonstrated that investigaton of the intrinsic heavy quark contributions looks very promising at the LHC in processes like $p p \rightarrow \gamma+c(b)+X$.
\end{abstract}

\section{Introduction}

The Large Hadron Collider (LHC) opens up new and unique kinematical regions with high accuracy for the investigation of the structure of the proton, in particular for the study of the parton distribution functions (PDFs). It is well known that the precise knowledge of the PDFs is very essential for the verification of the Standard Model and the search for New Physics.

Analyzing hadroproduction of so-called leading hadrons Brodsky et al. [1, 2] (about thirty years ago) has assumed co-existing of extrinsic and intrinsic contributions to the quark-gluon structure of the proton. The extrinsic (or ordinary) quarks and gluons are generated on a short time scale associated with large-transversemomentum processes. Their distribution functions satisfy the standard QCD evolution equations. The intrinsic quarks and gluons exist over a time scale which is independent of any probe momentum transfer. They can be associated with a bound-state (zero-momentum transfer regime) hadron dynamics and one believes they have a nonperturbative origin.

It was shown in [2] that the existence of intrinsic heavy quark pairs $c \bar{c}$, and $b \bar{b}$ within the proton state can be due to the virtue of gluon-exchange and vacuum-polarization graphs. On this basis, within the MIT bag model [3], the probability to find a five-quark component $|u u d c \bar{c}\rangle$ bound within the nucleon bag is nonzero and can be about $1-2 \%$.

Initially in $[1,2] \mathrm{S}$. Brodsky and coauthors proposed the existence of the 5-quark state $|u u d c \bar{c}\rangle$ in the proton. Later some other models were developed. One of them considered a quasi-two-body state $\bar{D}^{0}(u \bar{c}) \bar{\Lambda}_{c}^{+}(u d c)$ in the proton [4]. In order to not contradict the DIS HERA data

\footnotetext{
a. e-mail: bedny@jinr.ru

b. e-mail: mdemichev@jinr.ru

c. e-mail: gennady.lykasov@cern.ch

d. e-mail: stavreva@1psc.in2p3.fr

e. e-mail: mark.stockton@cern.ch
}

the probability to find the intrinsic charm (IC) in the proton (the weight of the relevant Fock state in the proton) was found to be less than 3.5\% [4]-[6]. The probability of finding intrinsic bottom state (IB) in the proton is suppressed by a factor of $m_{c}^{2} / m_{b}^{2} \simeq 0.1$ [7], where $m_{c} \simeq 1.3 \mathrm{GeV}$ and $m_{b}=4.2 \mathrm{GeV}$ are the current masses of the charm and bottom quarks. Therefore, the experimental search for a possible IC signal in $p p$ collisions at the LHC energies is more promising than the search for the IB contribution.

If the distributions of the intrinsic charm or bottom in the proton are hard enough and similar in the shape to the valence quark distributions (i.e. have valence-like form), then one expects the production of the charmed (bottom) mesons or charmed (bottom) baryons in the fragmentation region to be similar to the production of pions or nucleons (from the light quarks). However, the yield of this production depends on the probability to find intrinsic charm or bottom in the proton, but this amount looks too small. The PDFs that include the IC contribution in the proton have already been used in perturbative QCD calculations in [4][6].

In this paper, having in mind these considerations we give predictions for the LHC semi-inclusive $p p$ production of prompt photons accompanied by $c$-jets including the intrinsic charm component in the PDF.

For completeness, in these predictions the sea-like charm PDF [5] is also considered. As described in [5] this is a purely phenomenological scenario, where the intrinsic sea-like charm at the initial scale $Q_{0}=m_{c}$ is believed to be proportional to the light sea PDFs, i.e. $c(x)=\bar{c}(x) \sim$ $\bar{u}(x)+\bar{d}(x)$. This distribution tends to be larger at most $x$ values when compared to the ordinary charm distribution (CTEQ6.6M).

In the next section we analyze in detail the hard process of the real photon production in $p p$ collision at the LHC energies accompanied by the $c$-jet including the IC contribution in the proton. 


\section{Prompt photon and $c$-jet production}

Recently the investigation of prompt photon and $c(b)$ jet production in $p \bar{p}$ collisions at $\sqrt{s}=1.96 \mathrm{TeV}$ was carried out at the TEVATRON [8]-[10]. In particular, it was observed that the ratio of the experimental spectrum of the prompt photons, (accompanied by the $c$-jets) to the relevant theoretical expectation (based on the conventional PDF which ignored the intrinsic charm) increases with $p_{T}^{\gamma}$ up to factor about 3 when $p_{T}^{\gamma}$ reaches $110 \mathrm{GeV} / c$. Furthermore, taking into account the CTEQ66c PDF, which includes the IC contribution obtained within the BHPS model [1,2] one can increase this ratio up to 1.5 [11]. For the $\gamma+b$-jets $p \bar{p}$-production no enhancement in the $p_{T}^{\gamma}$-spectrum was observed at the beginning of the experiment $[8,10]$. However in 2012 the $\mathrm{D} \varnothing$ collaboration has confirmed observation of such an enhancement [9].

This intriguing observation stimulates our interest to look for a similar "IC signal" in $p p \rightarrow \gamma+c(b)+X$ processes at LHC energies. In Fig. 1 the distribution $d \sigma / d p_{T}^{\gamma}$ of prompt photons produced in the reaction $p p \rightarrow \gamma+c+X$ at $\sqrt{s}=8 \mathrm{TeV}$ is presented in the interval of the photon rapidity $1.52<\left|y_{\gamma}\right|<2.37$ and the $c$-jet rapidity $\left|y_{c}\right|<2.4$ The upper line (open circles) in the top of Fig. 1 is calculated with the CTEQ66c PDF and includes IC, while the lower line (dark circles) uses the CTEQ66 PDF where the charm PDF is radiatively generated only. The probability of the IC contribution is about $3.5 \%$ [6]. The ratio of the spectra with IC and without IC as a function of $p_{T}^{\gamma}$ is presented in the bottom of Fig. 1 the distribution $d \sigma / d p_{T}^{\gamma}$ of prompt photons produced in the reaction $p p \rightarrow \gamma+c+X$ at $\sqrt{s}=8 \mathrm{TeV}$ is presented in the interval of the photon rapidity $1.52<\left|y_{\gamma}\right|<2.37$ and the $c$-jet rapidity $\left|y_{c}\right|<2.4$.. including the radiation corrections for the initial (ISR) and final (FSR) states along with the multi-parton interactions (MPI) within PYTHIA8.

According to this figure, the IC signal can be about $180 \%-250 \%$ at $p_{T}^{\gamma} \simeq 150-200 \mathrm{GeV} / \mathrm{c}$ and the cross section is about $10-40 \mathrm{fb}$, which corresponds to about $1000-4000$ events in the $5 \mathrm{GeV} / \mathrm{c}$ bin at $L=20 \mathrm{fb}^{-1}$.

In Fig. 2 the differential cross-section $d \sigma / d p_{T}^{\gamma}$ calculated at NLO in the massless quark approximation as described in [11] is presented as a function of the transverse momentum of the prompt photon. The following cuts are applied : $p_{T}^{\gamma}>45 \mathrm{GeV}, p_{T}^{c}>20 \mathrm{GeV}$ with the $c$-jet rapidity in the interval $\left|y_{c}\right| \leq 2.4$ and the photon rapidity region $1.52<\left|y_{\gamma}\right|<2.37$.

The solid blue line represents the differential crosssection calculated with the radiatively generated charm PDF (CTEQ66), the dash-dotted green line uses as input the sea-like PDF (CTEQ66c4) and the dashed red line the BHPS PDF (CTEQ66c2). In the lower half of Fig. 2 the above distributions normalized to the distribution acquired using the CTEQ66 PDF and $\mu_{r}=\mu_{f}=\mu_{F}=p_{T}^{\gamma}$, are presented. The shaded yellow region, represents the scale dependence. The difference when using the BHPS IC PDFs is about $200 \%$ at $p_{T}^{\gamma} \sim 200 \mathrm{GeV}$ and increases almost up to $300 \%$ for $p_{T}^{\gamma} \sim 400 \mathrm{GeV}$. In this rapidity region the difference between the BHPS and sea-like spectra is clearly visible even as early as $p_{T}^{\gamma} \sim 200 \mathrm{GeV}$. However as in

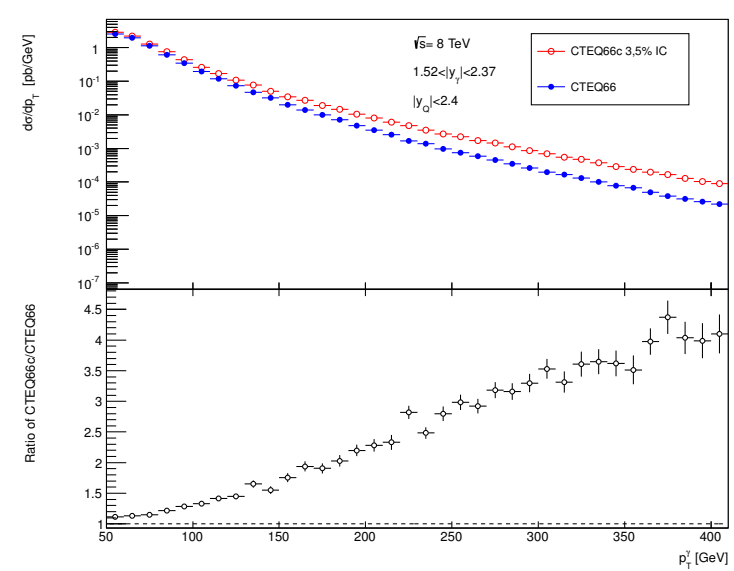

Figure 1. The distribution $d \sigma / d p_{T}^{\gamma}$ of prompt photons produced in the reaction $p p \rightarrow \gamma c X$ over the transverse momentum $p_{T}^{\gamma}$ integrated over $d y$ in the interval $1.52<\left|y_{\gamma}\right| 2.37,<\left|y_{c}\right|<2.4$ at $\sqrt{s}=8 \mathrm{TeV}$. The red open points correspond to the inclusion of the IC contribution in PDF CTEQ66c with the IC probability of about $3.5 \%$ [6]; the blue solid points is our calculation using the CTEQ66 without the IC contribution in the proton. The calculation was done within PYTHIA8 using the LO QCD and including the ISR, FSR and MPI.

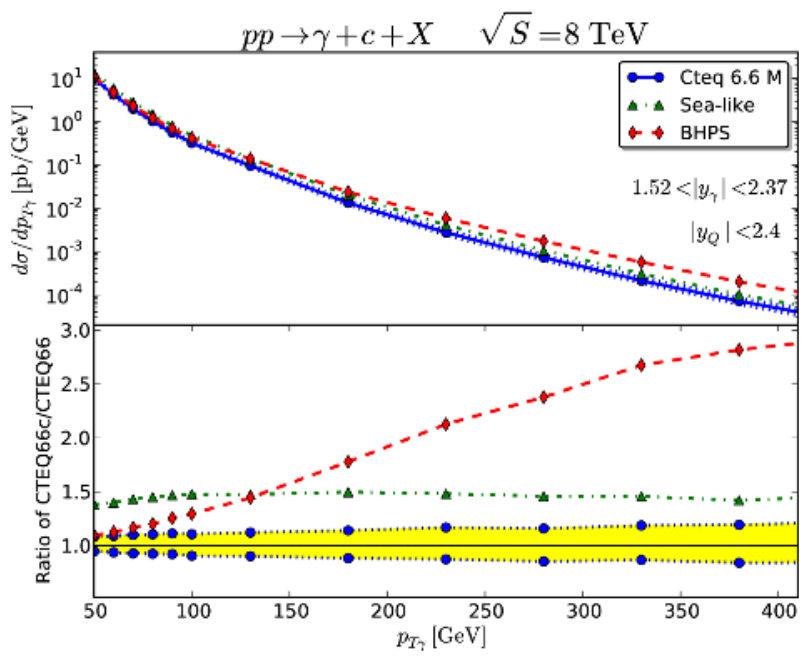

Figure 2. The $d \sigma / d p_{T}^{\gamma}$ distribution versus the transverse momentum of the photon for the process $p p \rightarrow \gamma+c+X$ at $\sqrt{s}=8 \mathrm{TeV}$ using CTEQ6.6M (solid blue line), BHPS CTEQ6c2 (dashed red line) and sea-like CTEQ6c4 (dash-dotted green line), for forward photon rapidity $1.52<\left|y_{\gamma}\right|<2.37$ (top). The ratio of these spectra with respect to the CTEQ6.6M (solid blue line) distributions (bottom). The calculation was done within the NLO QCD approximation without ISR, FSR and MPI.

Fig. 1 while the IC is more accentuated, the cross-section and hence the number of events is less than those for the photon central rapidity.

Note that comparing Fig. 1 to Fig. 2 one can see that both the LO QCD and NLO QCD cross-section results in approximately the same IC contribution, which increases when the photon transverse momentum grows. Nevertheless the values of the spectra calculated within the NLO 
QCD are larger by a factor of about 1.2 than the ones obtained within the LO QCD at $p_{T}^{\gamma}>100 \mathrm{GeV} / \mathrm{c}$ including the ISR, FSR and the MPI. Note that all the calculations presented in Figs. (1-2) were done for the isolated photons.

Therefore Figs 1,2 show that the IC signal could be visible at the LHC energies with both the ATLAS and CMS detectors in the process $p p \rightarrow \gamma+c+X$ when $p_{T}^{\gamma} \simeq$ $150 \mathrm{GeV} / \mathrm{c}$. In the region the IC signal dominates over the all non-intrinsic charm background with significance at a level of a factor of 2 (in fact 170\%).

\section{Conclusion}

In this paper we have shown that the possible existence of the intrinsic heavy quark components in the proton can be seen not only in the forward open heavy flavor production in $p p$-collisions (as it was believed before) but it can be visible also in the semi-inclusive $p p$-production of prompt photons and $c$-jets at rapidities $1.5<\left|y_{\gamma}\right|<2.4$, $\left|y_{c}\right|<2.4$ and large transverse momenta of photons and jets.

In the inclusive photon spectrum measured together with a c-jet a rather visible enhancement can appear due to the intrinsic charm (IC) quark contribution. In particular, it was shown that the IC contribution can produce much more events (factor 2 or 3 ) at $p_{T}^{\gamma}>150 \mathrm{GeV} / \mathrm{c}$ and forward $y_{\gamma}$ in comparison with the the relevant number expected in the absence of the IC. Furthermore, the same enhancement is also coherently expected in the transverse momentum, $p_{T}^{c}$, distribution of the $c$-jet measured together with the above-mentioned prompt photon in the $p p \rightarrow \gamma+c$ jet $+X$ process.

Searching for the signal of intrinsic charm in such processes is more pronounced than the search for the intrinsic bottom because the IB probability is, at least, in 10 times smaller than the IC probability in the proton.
Our predictions can be verified at the LHC by the ATLAS and CMS Collaborations. To this end further consideration of non-charm (light quarks) backgrounds for the discussed processes is mandatory.

\section{Acknowledgments}

We thank S.J. Brodsky and A.A. Glasov for extremely helpful discussions and recommendations by the study of this topic. The authors are grateful to H. Jung, A.V. Lipatov, V.A.M. Radescu, A. Sarkar and N.P. Zotov for very useful discussions and comments. This research was also supported by the RFBR grants No. 11-02-01538-a and No. 13-02001060.

\section{References}

[1] S. Brodsky, P. Hoyer, C. Peterson, N. Sakai, Phys.Lett. B 93, 451 (1980)

[2] S. Brodsky, C. Peterson, N. Sakai, Phys.Rev. D, 23, 2745 (1981)

[3] J.F. Donoghue, E. Golowich, Phys.Rev.D 15, 3421 (1977)

[4] J. Pumplin,Phys.Rev. D 73, 114015 (2006)

[5] J. Pumplin, H.L. Lai, W.K. Tung, Phys.Rev. D 75, 054029 (2007)

[6] P.M. Nadolsky, et al., Phys. Rev. D 78, 013004 (2008)

[7] M.V. Polyakov, A. Schafer, O.V. Teryaev, Phys.Rev. D 60, 051502 (1999)

[8] V.M. Abazov, et al., Phys.Rev.Lett. 102192002 (2009); arXiv :0901.0739 [hep-ex].

[9] V.M. Abazov, et al., Phys.Lett. B 714, 192002 (2012); arXiv :1203.5865 [hep-ex].

[10] T. Aaltonen, et al., Phys.Rev. D 81, 052006 (2010); arXiv :0912.3453 [hep-ex].

[11] T.P. Stavreva, and J.F. Owens, Phys.Rev. D 79, 054017 (2009); arXiv :0901.3791 [hep-ph]. 